\begin{tabular}{|c|c|}
\hline Title & Effect of A s preadsorption on InA s nanowire heteroepitaxy on $\mathrm{Si}(111)$ : A first-principles study \\
\hline Author(s) & Koga, Hiroaki \\
\hline Citation & $\begin{array}{l}\text { Physical review. B, Condensed matter and materials physics, 80(24), } 245302 \\
\text { https://doi.org/10.1103/Phy sRevB. } 80.245302\end{array}$ \\
\hline Issue Date & $2009-12$ \\
\hline Doc URL & http:/hdl.handle.net/2115/40002 \\
\hline Rights & (c) 2009 A merican Physical Society \\
\hline Type & article \\
\hline Note & 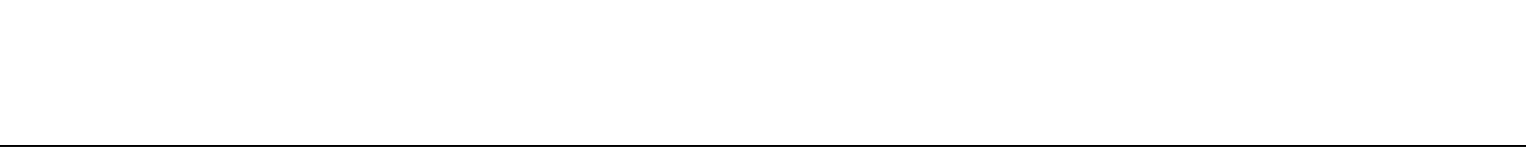 \\
\hline File Information & 2009K ogaPhysRevB.pdf \\
\hline
\end{tabular}

Instructions for use 


\title{
Effect of As preadsorption on InAs nanowire heteroepitaxy on Si(111): A first-principles study
}

\author{
Hiroaki Koga \\ Research Center for Integrated Quantum Electronics (RCIQE), Hokkaido University, North 13, West 8, Sapporo 060-8628, Japan
}

(Received 28 July 2009; revised manuscript received 3 November 2009; published 1 December 2009)

\begin{abstract}
Arsenic preadsorption has recently been found to be crucial for selective-area epitaxial growth of oriented III-V semiconductor nanowires on $\mathrm{Si}(111)$. To understand the effect of preadsorption on the heteroepitaxy, this first-principles study examines the structure of As-adsorbed Si(111) surfaces. Reconstruction models such as adatom, trimer, and dimer-adatom-stacking fault structures are found to be metastable. The stability of unreconstructed arsenide structure $(1 \times 1$-As $)$ is confirmed but the faulted and unfaulted domains of $1 \times 1$-As are found to be practically degenerate in energy. These domains can therefore coexist on a $\mathrm{Si}(111)$-As surface, and then epitaxial growth will be disrupted at domain boundaries where translational symmetry is broken. Indium adsorption on the $\mathrm{Si}(111)$-As surface, however, destabilizes unfaulted domains, thus assisting its transformation into a coherent surface that allows epitaxy. This effect is attributed to the interlayer covalent interactions induced by In $p$ electrons.
\end{abstract}

DOI: $10.1103 /$ PhysRevB.80.245302

PACS number(s): 68.43.Bc, 68.47.Fg, 61.72.uf, 61.72.uj

\section{INTRODUCTION}

Epitaxial growth of nanoscale structures differs from that of thin films in the size of the substrate surface on which they grow. Diminishing the substrate surface minimizes the effects due to lattice mismatch, allowing heteroepitaxial growth of mismatched systems, for example, III-V semiconductor nanowires on a Si substrate. ${ }^{1}$ It also excludes mesoscopic features such as steps and dislocations, leaving the atomic structure to play a major role. Clarifying the atomic structure of the substrate surface is therefore key to understanding the growth of nanostructures.

Recent experiments on selective-area vapor-phase epitaxy of III-V nanowires on $\mathrm{Si}^{2,3}$ which we discuss here, provide a salient example. Lately, the miniaturization of Si transistors is approaching a limit, and the continuing development of semiconductor electronics will most likely require integrating alternative channel materials such as III-V semiconductor nanowires onto $\mathrm{Si}$. The selective epitaxy, which allows free-standing nanowires to grow through lithographically created openings of an oxide mask covering a substrate, ${ }^{4}$ is a promising technique for achieving this integration. This method also enables efficient study of the effect of substrate surfaces on nanowire growth because mask openings serve as an ensemble of very small $(\sim 100 \mathrm{~nm})$ surfaces.

The orientations of the nanowires observed in the experiments ${ }^{2,3}$ signify the extent to which the nanowire heteroepitaxy is influenced by the atomic structure of the substrate surface. III-V semiconductors such as InAs and GaAs grow into vertical nanowires on $(\overline{1} \overline{1} \overline{1})$ and into tilted ones on (111) because $\langle\overline{1} \overline{1} \overline{1}\rangle$ is the preferential growth direction under nanowire growth conditions [Fig. 1(b)]. On a Si substrate, however, both vertical and tilted nanowires grow because inversion symmetry makes $(111)$ and $(\overline{1} \overline{1} \overline{1})$ equivalent. To increase the percentage of vertical InAs nanowires on $\mathrm{Si}$, the experimentalists ${ }^{2}$ tried exposing the substrate to arsine, thus turning the exposed $\mathrm{Si}(111)$ surfaces into the unreconstructed arsenide structure $(1 \times 1-\mathrm{As}),{ }^{5}$ which resembles the ideal $\operatorname{InAs}(\overline{1} \overline{1} \overline{1})$ surface (Fig. 1). Although this approach eventually proved successful, the results varied significantly

depending on the way the As preadsorption was carried out. First, the percentage of mask openings growing InAs nanowires (yield) was very low $(\sim 50 \%)$ when the substrate was exposed to arsine at high temperatures while cooling from thermal cleaning temperatures $\left(925^{\circ} \mathrm{C}\right)$ to the nanowire growth temperature $\left(540{ }^{\circ} \mathrm{C}\right)$. The yield improved to $\sim 70 \%$ when the substrate was exposed to arsine at a low temperature $\left(400{ }^{\circ} \mathrm{C}\right)$ and then heated to the nanowire growth temperature. The yield reached almost 100\% (95\% vertical, 5\% tilted) when the low-temperature As adsorption was followed by alternating cycles of In and As depositions [flow-rate modulation epitaxy (FME)] prior to nanowire growth. From these results, it was thought that complex $\mathrm{Si}(111)$-As reconstructions appeared at the high temperatures, disrupting epitaxial growth.

Clarifying the structure of the As-adsorbed surfaces is thus vital for understanding the nanowire heteroepitaxy. The detailed structure is however unclear from the vapor-phase experiments while $1 \times 1-$ As is the only phase found in ultrahigh-vacuum (UHV) studies. ${ }^{5-11}$ Although previous first-principles calculations have shown that the $\sqrt{3} \times \sqrt{3} T_{4}$ adatom, ${ }^{12-14} \sqrt{3} \times \sqrt{3}$ trimer, ${ }^{15}$ and $2 \times 1$ zigzag chain ${ }^{15}$ structures are metastable to $1 \times 1-\mathrm{As}$, the possibility of composite

(a)

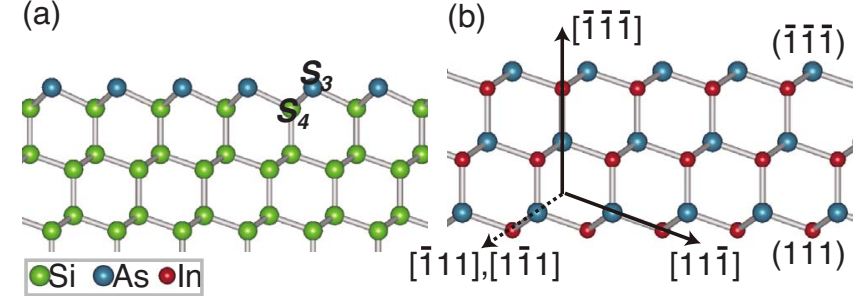

FIG. 1. (Color online) Side view (from $[\overline{1} 10])$ of (a) $1 \times 1$-As model and (b) ideal $\operatorname{InAs}(\overline{1} \overline{1} \overline{1})$ and (111); not to scale. The arrows indicate the four equivalent directions of $\langle\overline{1} \overline{1} \overline{1}\rangle$, namely, [ $\overline{1} \overline{1} \overline{1}]$, $[11 \overline{1}],[\overline{1} 11]$, and $[1 \overline{1} 1]$. Note that the last two directions (the dotted arrow) are not on the plane of the figure; they look identical as projected onto the plane but are actually separated by a tetrahedral angle. 
structures involving adatoms, rest atoms, and stacking faults has not been excluded.

An alternative explanation for the low yield is the coexistence of energetically degenerate domains of $1 \times 1$-As. Differing merely in whether As atoms occupy hcp or fcc sites, faulted and unfaulted domains of $1 \times 1$-As are likely to be nearly degenerate in energy. The coexistence of the two domains will disrupt epitaxial growth because translational symmetry is broken at the domain boundaries. Then, the observed improvement in yield by FME suggests that the degeneracy is removed by In deposition, the first step in the FME. Thus, it is useful to calculate the relative stability of the two domains and to examine how the relative stability is affected by In. Understanding the effect of adsorbates on stacking faults is also useful for predicting the occurrence of stacking faults (and polytypism) in semiconductor nanowires, an active topic in many theoretical investigations. ${ }^{16-22}$

This first-principles study therefore examines $\mathrm{Si}(111)$-As surface geometries and the effect of In on $1 \times 1$-As. The rest of this paper is organized as follows. First, the detail of density-functional theory ${ }^{23}$ (DFT) calculations is described and the accuracy of DFT results is discussed (Sec. II). Then, $\mathrm{Si}(111)$-As reconstruction models are examined and the stability of $1 \times 1-\mathrm{As}$ is confirmed (Sec. III A). Thereafter, the degeneracy and coexistence of faulted and unfaulted domains of $1 \times 1$-As, not As-induced reconstructions, is suggested as a cause disrupting epitaxial growth (Sec. III B). Furthermore, In deposition is shown to relieve this degeneracy problem by destabilizing unfaulted domains. The results are summarized in Sec. IV.

\section{METHOD}

Total energies and optimized geometries were calculated within generalized gradient approximation ${ }^{24}$ (GGA) of DFT by a first-principles molecular-dynamics code STATE, which has been applied to various systems ${ }^{25-28}$ including $\mathrm{Si}$ surfaces adsorbed with metal and a group-V element. ${ }^{28}$ KohnSham equations ${ }^{29}$ were solved by expansion into plane waves up to the cutoff energy of 16 Ry. Interactions between valence electrons and ion cores were described by normconserving pseudopotentials. ${ }^{30}$ The $\mathrm{Si}(111)$ substrate was modeled by a periodic slab of eight atomic layers [i.e., four fcc(111) planes], terminated in $\mathrm{H}$ at the bottom. The $\mathrm{H}$ terminators as well as the two bottom $\mathrm{Si}$ layers were frozen during geometrical optimization. The unit cells (and $k$-point meshes) used were $1 \times 1 \quad(8 \times 8), \sqrt{3} \times \sqrt{3}(4 \times 4), 2 \times 1$ $(4 \times 8), 2 \times 2(4 \times 4)$, and $7 \times 7(2 \times 2)$, where $1 \times 1$ denotes the primitive cell of the ideal $\mathrm{Si}(111)$ surface. Three classes of $\mathrm{Si}(111)$-As models were calculated. The first was those (see Figs. 3 and 4 below) derived from $\operatorname{Si}(111)$ models $-\sqrt{3} \times \sqrt{3}$ adatom, $2 \times 2$ adatom, and $7 \times 7$ dimeradatom-stacking fault ${ }^{31}$ (DAS) — by substituting As atoms for $\mathrm{Si}$ adatoms and rest atoms; the adatoms may sit on top of the second-layer $\mathrm{Si}\left(T_{4}\right)$ or above the centers $\left(H_{3}\right)$ of hexagonal rings, so both configurations were examined. The replacement of unsaturated Si dangling bonds with saturated As ones was expected to stabilize these monomer-terminated models. The second was those (see Fig. 5 below) derived from $\mathrm{Si}(111)-\mathrm{Sb}$ models $-\sqrt{3} \times \sqrt{3}$ trimer $^{32}$ and $2 \times 1$ zigzag chain $^{33}$ - by substituting As for $\mathrm{Sb}$. These full-coverage models were included for thoroughness although localdensity approximation ${ }^{34}$ (LDA) results were available in the literature. ${ }^{15}$ The third was polytypes of $1 \times 1$-As [see Fig. 7 (a) below], differing in the presence or absence of a stacking fault beneath the As layer. In addition to $\mathrm{Si}(111)$-As models, the $\operatorname{Si}(111) 1 \times 1$ relaxed, $2 \times 1$ Pandey chain, ${ }^{35}$ and $7 \times 7$ DAS structures were also calculated using the above conditions, to be used as a reference.

The stability of a $\mathrm{Si}(111)$-As model is determined by formation energy $\Omega$, calculated as

$$
\Omega=\Delta E-n_{\mathrm{Si}} \mu_{\mathrm{Si}}-n_{\mathrm{As}} \mu_{\mathrm{As}},
$$

where $\Delta E$ is the total energy measured from the reference [chosen to be the $\mathrm{Si}(111) 1 \times 1$ relaxed geometry], and $n_{\mathrm{Si}}$ and $n_{\mathrm{As}}$ are the changes in numbers of $\mathrm{Si}$ and As atoms, respectively; because the $\mathrm{Si}$ chemical potential $\mu_{\mathrm{Si}}$ is at the bulk level when the surface is in equilibrium, the formation energy becomes a linear function of the As chemical potential $\mu_{\mathrm{As}}$. It should be noted that vibrational and entropy contributions to the free energy of a solid surface are not included here. ${ }^{36}$

Although DFT calculations suffice to predict the stability of $\mathrm{Si}(111)$-As models, it is useful to interpret the results using a simple qualitative model. Most structures discussed in this paper are electronically saturated ones, which are bound mainly by two-center two-electron bonds. We thus express the binding energy of such a structure as a sum of bond energies $\left(E_{\mathrm{As}-\mathrm{Si}}, E_{\mathrm{Si}-\mathrm{Si}}\right.$, and $\left.E_{\mathrm{As}-\mathrm{As}}\right)$, neglect variations due to stress, and only consider the dependence of $E_{\mathrm{As}-\mathrm{Si}}$ on the site $(\alpha)$ occupied by As,

$$
E_{\text {bind }}=N_{\mathrm{Si}-\mathrm{Si}} E_{\mathrm{Si}-\mathrm{Si}}+N_{\mathrm{As}-\mathrm{As}} E_{\mathrm{As}-\mathrm{As}}+\sum_{\alpha} N_{\mathrm{As}-\mathrm{Si}}^{\alpha} E_{\mathrm{As}-\mathrm{Si}}^{\alpha},
$$

here $N_{\mathrm{Si}-\mathrm{Si}}, N_{\mathrm{As}-\mathrm{As}}$, and $N_{\mathrm{As}-\mathrm{Si}}$ are the numbers of $\mathrm{Si}-\mathrm{Si}, \mathrm{As}-$ $\mathrm{As}$, and As-Si bonds, respectively. The variation in $E_{\mathrm{As}-\mathrm{Si}}$ mainly depends on the degree to which $\mathrm{Si}$ dangling-bond (DB) orbitals are oriented toward As. The DB orientations can be found by an H-substitution test: an As atom is deleted from a $\mathrm{Si}(111)$-As geometry, resulting $\mathrm{Si} \mathrm{DBs}$ are terminated with one $\mathrm{H}$ atom each, and then only the $\mathrm{H}$ atoms are allowed to find optimum positions. The H-Si bonds thus obtained indicate the DB orientations.

Discussion on the accuracy of the present calculations is in order. First, the calculated lattice constants of $\mathrm{Si}$, InAs, and $\mathrm{GaAs}$ (5.47, 6.04, and 5.66, respectively) agree with those in the literature ${ }^{37}$ within $1 \%$; the cohesive energies are also reproduced $(4.53,3.15$, and 3.30 eV/atom, respectively) to within $3 \%$. Second, the calculation conditions are sufficient for the convergence of energy differences; the energy difference between $T_{4}$ and $H_{3}$ adatom geometries changes little $(\sim 4 \mathrm{meV})$ even if we use $6 \times 6 k$ points, 25 Ry cutoff, or a ten-layer slab. Third, the total energy per $1 \times 1$ changes little with cell size $(\sim 2 \mathrm{meV}$ for $1 \times 1-\mathrm{As})$. This proportionality allows arithmetic operations on energies given by various cells. Finally, the geometries obtained in earlier firstprinciples calculations ${ }^{12}$ are well reproduced (Table I). 
TABLE I. Structural parameters for Si(111)-As geometries. $b$ (pm) denotes distance between first neighbors of As. $\phi$ and $\theta\left(^{\circ}\right)$ denote Si-As-Si angles. LDA values are from Ref. 12.

\begin{tabular}{lccc}
\hline \hline & \multicolumn{2}{c}{$\sqrt{3} \times \sqrt{3} T_{4}(\mathrm{As})$} & $1 \times 1-\mathrm{As}$ \\
\cline { 2 - 3 } & $b$ & $\phi$ & $\theta$ \\
\hline Present & 335 & 80.9 & 102.9 \\
LDA & 332 & 81.8 & 104.5 \\
\hline \hline
\end{tabular}

\section{RESULTS AND DISCUSSION}

\section{A. Reconstructions of $\mathrm{Si}(111)$-As}

The variation in formation energy $\Omega$ with As chemical potential $\mu$ is shown in Fig. 2. This diagram shows that all the lines of $\mathrm{Si}(111)$-As reconstructions are bounded below by either the $7 \times 7$ DAS or $1 \times 1$-As lines. This confirms that $1 \times 1$-As is the only stable phase of the $\mathrm{Si}(111)$-As surface. The diagram also shows that $T_{4}$ is more stable than $H_{3}$ for both $\sqrt{3} \times \sqrt{3}$ and $2 \times 2$ models. In addition, energetic stability improves as we move from $\sqrt{3} \times \sqrt{3} T_{4}$ As-trimer to $2 \times 1$ zigzag chain to $1 \times 1-$ As, in agreement with earlier calculations. ${ }^{15}$ In the following, we analyze these trends mainly in terms of bond energies and Si DB orientations.

We begin with discussion of the relative stability of $T_{4}$ and $H_{3}$. Figure 2 shows that the $\sqrt{3} \times \sqrt{3} T_{4}$ As-adatom geometry is $0.22 \mathrm{eV} / 1 \times 1$ more stable than its $H_{3}$ counterpart [Fig. 3(a)]. This result is similar to that of group-III adatoms (Al, Ga, and $\mathrm{In})$ on $\mathrm{Si}(111)$, for which it was suggested that the substrate in $T_{4}$ deforms so that $\mathrm{Si}$ DBs point more di-

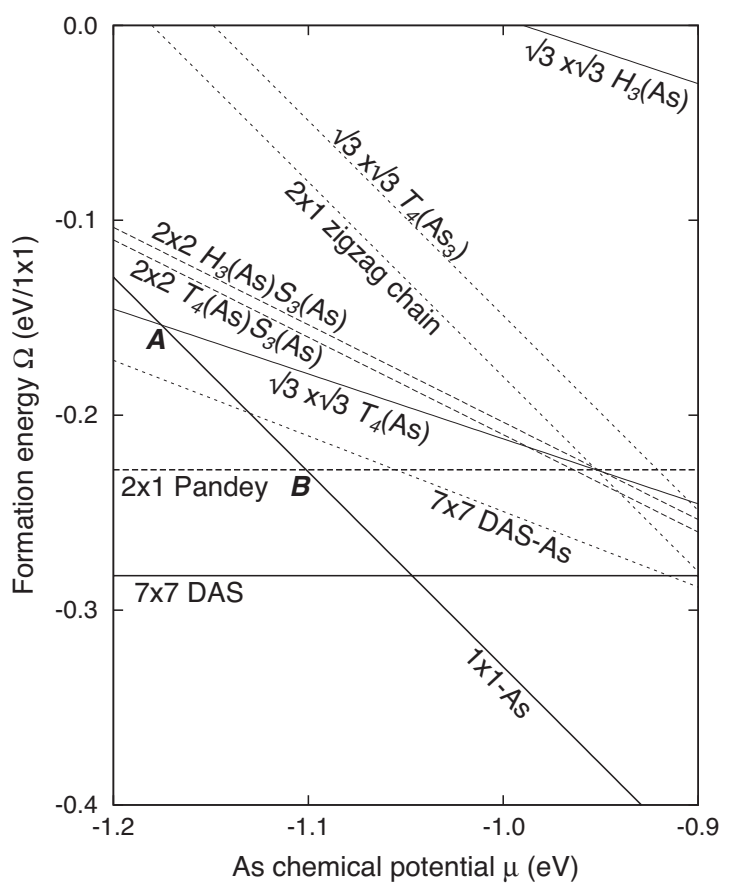

FIG. 2. Variation in formation energy $\Omega$ with As chemical potential $\mu$. The zero of $\mu$ is set at the As bulk level. Points $\mathrm{A}$ and $\mathrm{B}$ denote the intersections of the $1 \times 1$-As line with the $\sqrt{3} \times \sqrt{3}$ $T_{4}$ (As) and $2 \times 1$-Pandey lines, respectively (see text). (a) $\sqrt{3} \times \sqrt{3}$ adatom
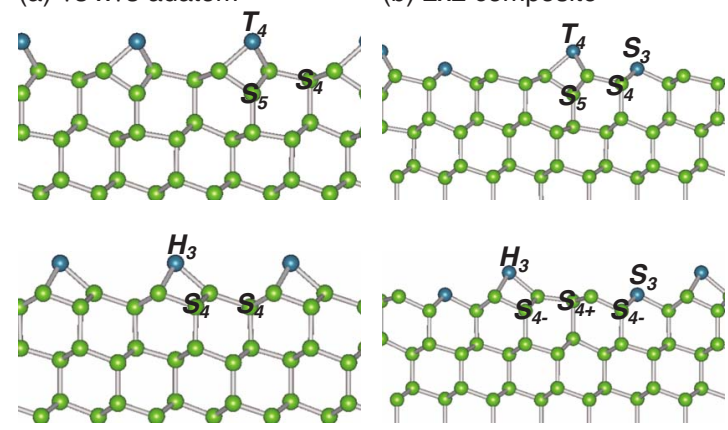

FIG. 3. (Color online) (a) $\sqrt{3} \times \sqrt{3}$ adatom and (b) $2 \times 2$ composite geometries; side view from $[\overline{1} 10]$.

rectly to the adatom. ${ }^{38}$ This can be seen in the optimized geometries shown in Fig. 3(a): the Si atoms located directly below $T_{4}$ adatoms $\left(S_{5}\right)$ descend by $51 \mathrm{pm}$ from the bulk position while the rest of the second-layer Si atoms $\left(S_{4}\right)$ ascend by $28 \mathrm{pm}$, thus reorienting the Si DBs toward As. Such movement is not allowed in $H_{3}$ because all the second-layer $\mathrm{Si}$ atoms $\left(S_{4}\right)$ are symmetrically equivalent. The $\mathrm{H}$-substitution test (Sec. II) accordingly finds that the Si DBs tilt $14.1^{\circ}$ from normal in $T_{4}$ but remain vertical in $H_{3}$.

The energy difference between $T_{4}$ and $H_{3}$ drastically decreases to $6 \mathrm{meV} / 1 \times 1$ if we move to $2 \times 2$ composite models [Fig. 3(b)], which contain both As adatoms $\left(T_{4}\right.$ and $\left.H_{3}\right)$ and rest atoms $\left(S_{3}\right)$. The H-substitution test accordingly finds that the degree of reorientation is similar in $T_{4}$ and $H_{3}\left(14.4^{\circ}\right.$ and $13.4^{\circ}$ from normal, respectively). The reorientation is allowed in $2 \times 2 \mathrm{H}_{3}$ because the first neighbors of $S_{3}\left(S_{4-}\right)$ and the rest of the second-layer $\mathrm{Si}\left(S_{4+}\right)$ are no longer equivalent; as can be seen in Fig. 3(b), $S_{4-}$ descends by 16 pm while $S_{4+}$ ascends by $52 \mathrm{pm}$.

Next, we discuss the metastability of adatom-induced reconstructions. The metastability of the $2 \times 2 T_{4}$ composite geometry can be seen in that its line (Fig. 2) is bounded below by the lines of $\sqrt{3} \times \sqrt{3} T_{4}$ (As) and $1 \times 1$-As, namely, that the former line is above point $A$ where the latter two intersect. Equivalently, the composition reaction

$$
\sqrt{3} \times \sqrt{3} T_{4}(\mathrm{As})+1 \times 1-\mathrm{As} \rightarrow 2 \times 2 T_{4}(\mathrm{As}) S_{3}(\mathrm{As})
$$

is energetically unfavorable (by $0.03 \mathrm{eV} / 1 \times 1$ ). Three reasons are conceivable for the lack of energy gain. First, As DBs are saturated on both sides, so there would be no energy gain associated with electron transfer. Second, both $\sqrt{3} \times \sqrt{3}$ $T_{4}(\mathrm{As})$ and $1 \times 1$-As are under tensile stress ${ }^{12}$ so the composition would not cancel the stress. Third, there is a dilemma about the position of the $S_{4}$ atom. This atom ascends by 28 pm in $\sqrt{3} \times \sqrt{3} T_{4}$ (As) [Fig. 3(a)] but descends by 5 pm in $1 \times 1$-As [Fig. 1(a)], so it cannot be in a position favorable to both adatoms and rest atoms in the composite geometry.

Similarly, the $\sqrt{3} \times \sqrt{3} T_{4}$ (As) line is $0.05 \mathrm{eV} / 1 \times 1$ above point $B$, where the $1 \times 1$-As and $2 \times 1$-Pandey lines intersect (Fig. 2), so the decomposition

$$
\sqrt{3} \times \sqrt{3} T_{4}(\text { As }) \rightarrow 1 \times 1-\text { As }+2 \times 1 \text {-Pandey }+\mathrm{Si},
$$

which relocates an As adatom to a rest-atom position, is energetically favorable. This can be discussed in terms of bond 


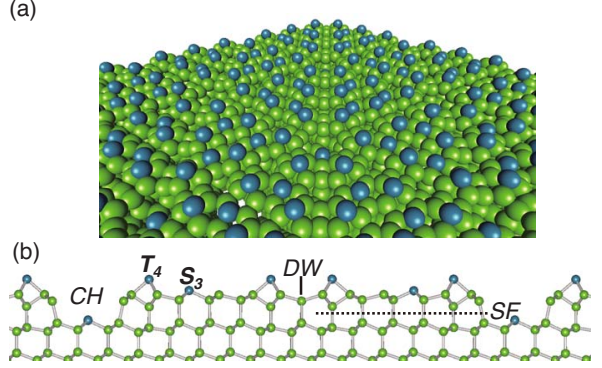

FIG. 4. (Color online) $7 \times 7$ DAS-As. (a) Perspective. (b) Side view from [110]; corner hole, dimer wall, and stacking fault are indicated.

energies because, unlike the decomposition involving $7 \times 7$ DAS, ${ }^{12-14}$ all the structures here are electronically saturated. Noting that each As atom is bonded to three $\mathrm{Si}$ atoms and that each $\mathrm{Si}$ atom has four bonds (this is so in bulk $\mathrm{Si}$ and also in the Pandey chain if we count $\pi$ bonds), we count three $\mathrm{As}-\mathrm{Si}$ bonds per $\mathrm{As}$ atom and four $\mathrm{Si}-\mathrm{Si}$ bonds per $\mathrm{Si}$ atom, reduce the Si-Si count by three for each As atom, and then correct for double counting. From this it follows that the bond counts are uniquely determined by the numbers of $\mathrm{Si}$ and As atoms. Moreover, the reaction conserves the bond counts because the numbers are balanced on both sides of the reaction. The energy gain thus reduces to that $\left(3 E_{\mathrm{As}-\mathrm{Si}}^{S_{3}}\right.$ $\left.-3 E_{\mathrm{As}-\mathrm{Si}}^{T_{4}}\right)$ due to the relocation of one As atom. Note that $E_{\mathrm{Si}-\mathrm{Si}}$ does not enter this expression although the formation of $1 \times 1$-As involves substitution for $\mathrm{Si}$. Within the bondenergy model, therefore, the metastability of the adatom structure simply reflects that an As rest atom's As-Si bonds are stronger than an adatom's.

The rest atom's stronger bonding can be seen in the geometry of $1 \times 1$-As [Fig. 1(a)]. The similarity in atomic radii (118 and $121 \mathrm{pm}$ ) between $\mathrm{As}$ and $\mathrm{Si}$, as well as the nearly $s p^{3}$-like bonding of As, permits the As rest atom to occupy a bulklike position toward which $\mathrm{Si} \mathrm{DBs}$ are oriented. The $\mathrm{H}$-substitution test indeed indicates that the misorientation of As-Si from Si DB is very small $\left(2.1^{\circ}\right)$ in $1 \times 1-A s$ but is comparatively large $\left(34.4^{\circ}\right)$ in $\sqrt{3} \times \sqrt{3} T_{4}(\mathrm{As})$. This is also reflected in the As-Si bond length, which is much shorter in $1 \times 1$-As than in the adatom structure (248 vs $259 \mathrm{pm})$.

The rest atom's stronger bonding $\left(E_{\mathrm{As}-\mathrm{Si}}^{S_{3}}>E_{\mathrm{As}-\mathrm{Si}}^{T_{4}}\right)$ also contributes to the stability of the As-substituted $7 \times 7$ DAS structure (Fig. 4). Figure 2 shows that the $7 \times 7$ DAS-As line is $0.03 \mathrm{eV} / 1 \times 1$ below point $A$ where the $\sqrt{3} \times \sqrt{3} T_{4}(\mathrm{As})$ and $1 \times 1-$ As lines intersect. That is, the composition reaction

$$
\begin{aligned}
15 \cdot \sqrt{3} & \times \sqrt{3} T_{4}(\mathrm{As})+4 \cdot 1 \times 1-\mathrm{As} \rightarrow 7 \times 7 \text { DAS-As } \\
+ & 11 \mathrm{Si}
\end{aligned}
$$

is energetically favorable. This reaction relocates three As adatoms to more stable rest-atom positions while conserving the bond counts for the reasons given above. The energy gain within the bond-energy model is thus positive $\left(9 E_{\mathrm{As}-\mathrm{Si}}^{S_{3}}\right.$ $\left.-9 E_{\mathrm{As}-\mathrm{Si}}^{T_{4}}>0\right)$, qualitatively consistent with the DFT prediction. On the other hand, the $7 \times 7$ DAS-As line is $0.02 \mathrm{eV} / 1 \times 1$ above point $B$ where the $1 \times 1-\mathrm{As}$ and (a)

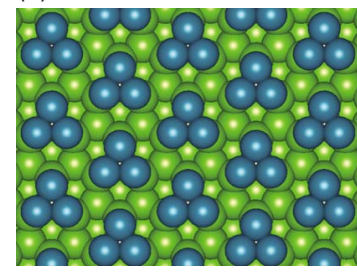

(b)

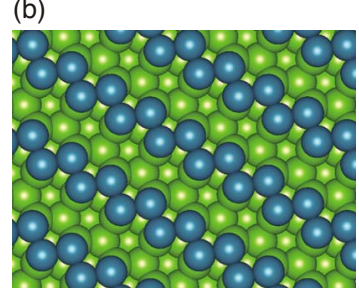

FIG. 5. (Color online) As monolayer on $\mathrm{Si}$ (111) (plan view). (a) $\sqrt{3} \times \sqrt{3} T_{4}$ As trimer. (b) $2 \times 1$ As chain.

$2 \times 1$-Pandey lines intersect (Fig. 2). That is, the decomposition reaction

$7 \times 7$ DAS-As $\rightarrow 19 \cdot 1 \times 1-$ As $+15 \cdot 2 \times 1$-Pandey $+4 \mathrm{Si}$

is energetically favorable. This reaction relocates 12 As adatoms to rest-atom positions without changing the bond counts. The energy gain within the bond-energy model is thus positive $\left(36 E_{\mathrm{As}-\mathrm{Si}}^{S_{3}}-36 E_{\mathrm{As}-\mathrm{Si}}^{T_{4}}>0\right)$, and hence the rest atom's stronger bonding actually favors the decomposition of the DAS-As structure.

Next, we discuss surfaces covered with an As monolayer. Optimized geometries for the $\sqrt{3} \times \sqrt{3} T_{4}$ As-trimer and 2 $\times 1$ As-chain structures are shown in Fig. 5. Although these isomers are equal in the number of each type of bonds and similar in As-As bond length (254 and $259 \mathrm{pm}$ ), the AsAs-As angle $\left(96.8^{\circ}\right)$ of the chain is much closer to the $p^{3}$-bonding angle $\left(90^{\circ}\right)$ favored by group-V atoms. Accordingly, the chain structure is found to be $0.03 \mathrm{eV} / 1 \times 1$ lower in energy than the trimer structure (Fig. 2). This agrees with that $(0.06 \mathrm{eV} / 1 \times 1)$ obtained in an earlier calculation ${ }^{15}$ to within the difference between GGA and LDA.

The chain structure is metastable to $1 \times 1$-As: the transformation

$$
2 \times 1 \text { As chain } \rightarrow 2 \cdot 1 \times 1-\mathrm{As}+2 \mathrm{Si}
$$

is favorable by $0.15 \mathrm{eV} / 1 \times 1$. The transformation trades two $\mathrm{Si}-\mathrm{Si}$ and two As-As bonds for four As-Si bonds while causing little change in As-Si bond length (from 247 to 248 $\mathrm{pm}$ ), indicating negligible variation in As-Si bond energy. Thus, the energy gain within the bond-energy model is $4 E_{\mathrm{As}-\mathrm{Si}}-2 E_{\mathrm{Si}-\mathrm{Si}}-2 E_{\mathrm{As}-\mathrm{As}}$, which becomes positive if the As-Si bond strength exceeds the average strength of As-As and $\mathrm{Si}-\mathrm{Si}$ bonds. This agrees with chemical intuition: similarity in atomic radii (118 and 121 p.m.) of As and Si allows overlap between orbitals and hence covalent bonding while an electronegativity difference (1.90 vs 2.18$)$ strengthens the heteronuclear bond by adding ionicity to it.

Finally, we ascertain that As adsorption stops at one monolayer. Consider $\sqrt{3} \times \sqrt{3}$-As $T_{4}(\mathrm{As})$ and $T_{4}\left(\mathrm{As}_{3}\right)$ models (Fig. 6). These models have As coverages of 1.33 and 2.0, respectively, with their substitutional monolayer and excess adsorbates. DFT calculations find that segregations into $1 \times 1-$ As and bulk As

$$
\sqrt{3} \times \sqrt{3}-\mathrm{As} T_{4}(\mathrm{As}) \rightarrow 3 \cdot 1 \times 1-\mathrm{As}+\mathrm{As}
$$


(a)
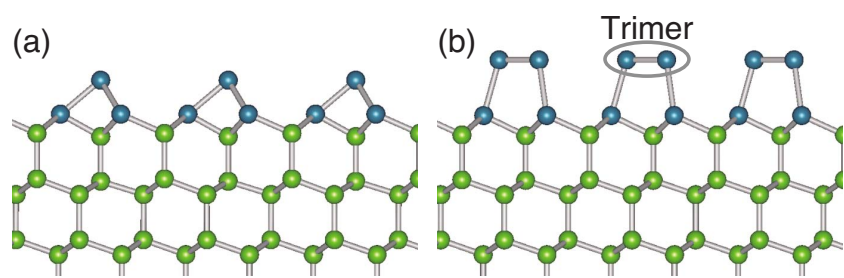

FIG. 6. (Color online) Excess As adsorption (side view from [110]): (a) $\sqrt{3} \times \sqrt{3}-\mathrm{As} T_{4}$ (As) and (b) $T_{4}\left(\mathrm{As}_{3}\right)$.

$$
\sqrt{3} \times \sqrt{3}-\mathrm{As}_{4} T_{4}\left(\mathrm{As}_{3}\right) \rightarrow 3 \cdot 1 \times 1-\mathrm{As}+3 \text { As }
$$

are extremely favorable by 2.95 and $1.87 \mathrm{eV}$ (per formula unit), respectively. As long as As chemical potential is below the bulk level, the segregated As will evaporate, limiting As adsorption to one monolayer. This is consistent with the experimental observation ${ }^{5}$ that excess As can be removed by annealing at a low temperature $\left(200{ }^{\circ} \mathrm{C}\right)$.

\section{B. Faulted and unfaulted domains of $1 \times 1-A s$}

We have seen that the unreconstructed $1 \times 1$-As structure is the stable phase of $\mathrm{Si}(111)$-As. However, DFT calculations also find that faulted and unfaulted models [Fig. 7(a)] of $1 \times 1$-As are practically degenerate in energy, differing by a mere $2 \mathrm{meV} / 1 \times 1$. The faulted and unfaulted domains can therefore coexist on the same surface, possibly separated by dimer walls as in the DAS structure. Epitaxial growth on such a multidomain surface will be difficult because translational symmetry is broken at the domain boundaries. This is consistent with the experimental observation ${ }^{2}$ that the yield of InAs nanowires is low if they are grown immediately after As adsorption. One way to avoid this problem is to wait for domains to merge through elimination of energetically unfavorable domain walls. Indeed, a medium-energy ionscattering experiment ${ }^{9}$ indicates that faulted domains are absent from $\mathrm{Si}(111)$-As surfaces prepared by UHV methods.

The degeneracy problem can also be relieved by In deposition. Nanowire growth experiments ${ }^{2}$ have found that the

(a) $1 \times 1-$ As
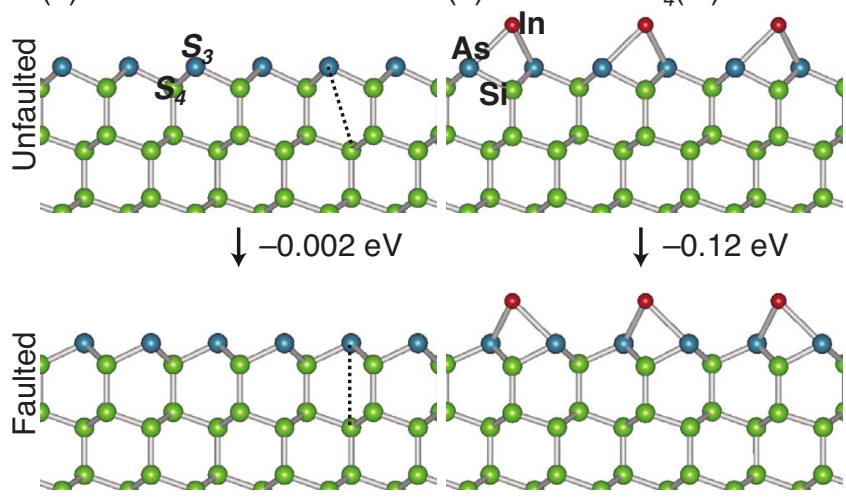

FIG. 7. (Color online) Faulted and unfaulted models of (a) $1 \times 1$-As and (b) $\sqrt{3} \times \sqrt{3}$-As $T_{4}(\mathrm{In})$; side view from [110]. Stacking fault energies per reconstruction cell are also shown. The dotted lines indicate the registration of the As layer with the fourth layer.
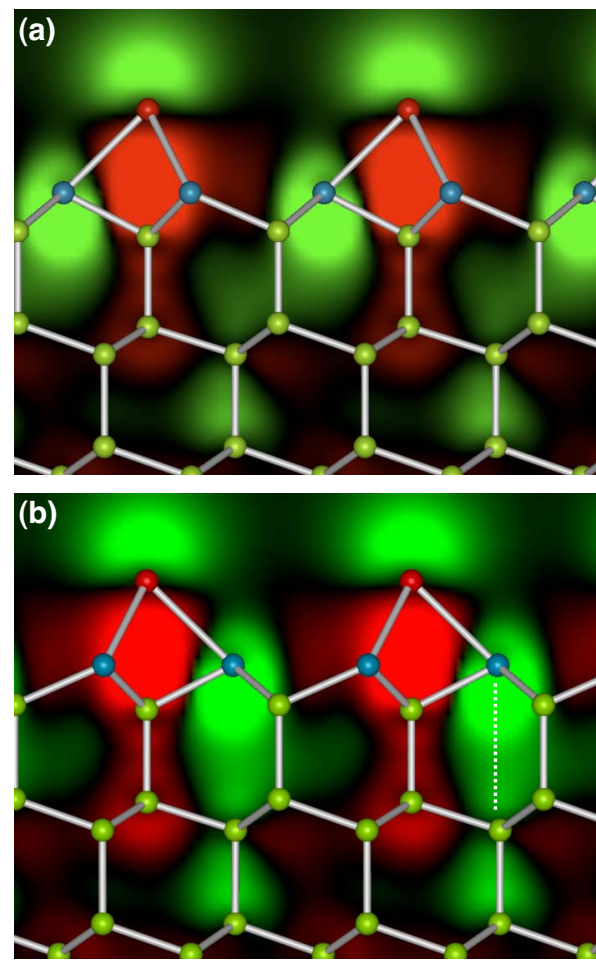

FIG. 8. (Color online) $\Gamma$-point orbitals belonging to the partially occupied band of the (a) unfaulted and (b) faulted $\sqrt{3} \times \sqrt{3}-\mathrm{As}$ $T_{4}(\mathrm{In})$ models [plot of the real part of orbital function on (110)]. The dotted line indicates the interlayer interaction discussed in the text.

yield of InAs nanowires improves to almost $100 \%$ when the $\mathrm{Si}(111)$-As surface is capped by InAs FME, starting with In deposition. Thus, the effect of In on the degeneracy is worth examining. For the $\sqrt{3} \times \sqrt{3}$-As $T_{4}(\mathrm{In})$ adatom geometry [Fig. $7(\mathrm{~b})]$, the faulted model is found to be $0.12 \mathrm{eV}$ lower in energy than the unfaulted one. ${ }^{39}$ A similar energy difference, $0.11 \mathrm{eV}$ in favor of the faulted model, is found for the $2 \times 2$-As $T_{4}$ (In) adatom geometry, indicating that it is In adatoms, rather than their arrangement into a particular pattern, that have the effect of stabilizing the unfaulted domains. Thus, at the early stage of In deposition, before the nucleation of an In layer, In adatoms facilitate the merging of the domains into a coherent surface that allows epitaxy. It also follows that a stacking fault will occur at the interface between the Si substrate and a nanowire grown in this manner.

The mechanism behind the stabilization of faulted domains is attributable to interlayer covalent interactions induced by In. In the faulted model, the As layer can interact with the fourth Si layer because they are in register with each other [Fig. 7(a)]. The interlayer interaction will be weak in the unfaulted model because the two layers are staggered. The energy difference would nevertheless be small in electronically saturated structures such as $1 \times 1$-As because there is no excess electron to participate in covalent interactions. When In is deposited on $1 \times 1$-As, however, unpaired In $p$ electrons become available for the covalent interactions. Indeed, the plot [Fig. 8(b)] of the $\Gamma$-point orbital belonging to the partially occupied band of the faulted model shows the hybridization of As $s$-like and Si $d_{z^{2}}$-like components be- 
tween the layers; in the unfaulted model, by contrast, these components are unable to overlap head-on [Fig. 8(a)]. This mechanism implies that other group-III elements such as $\mathrm{Ga}$ also stabilize faulted domains. The faulted model of $\sqrt{3} \times \sqrt{3}-\mathrm{As} T_{4}(\mathrm{Ga})$ is accordingly found to be $0.12 \mathrm{eV}$ more stable than its unfaulted counterpart.

\section{SUMMARY}

In summary, we have examined $\mathrm{Si}(111)$-As surface geometries at the GGA level and confirmed the stability of $1 \times 1$-As. Adatom, DAS-As, zigzag chain, and trimer structures are metastable and will transform into $1 \times 1$-As and clean $\mathrm{Si}(111)$. Formal analyses using a bond-energy model have shown that the stability of $1 \times 1$-As simply reflects two intuitive facts: (i) an As rest atom's As-Si bonds are stronger than an adatom's and (ii) heteronuclear bonds (As-Si) are stronger than homonuclear bonds (As-As and $\mathrm{Si}-\mathrm{Si}$ ). How- ever, the faulted and unfaulted domains of $1 \times 1$-As have been found practically degenerate in energy, so epitaxial growth can be disrupted by their coexistence. Indium adatoms on this surface stabilize the faulted domains by inducing interlayer covalent interactions, facilitating the merging of the domains. The In deposition after As adsorption thus assists the formation of coherent surfaces that allow epitaxial growth of InAs nanowires on Si.

\section{ACKNOWLEDGMENTS}

The author thanks K. Tomioka for useful discussions about nanowire growth experiments. The DFT calculations were run on the computers of the Information Technology Center, the University of Tokyo, and on those of the Research Institute for Information Technology, Kyushu University. This work was in part supported by JSPS under Grant No. 21860001.
${ }^{1}$ T. Mårtensson, C. P. T. Svesson, B. A. Wacaser, M. W. Larsson, W. Seifert, K. Deppert, A. Gustafsson, L. R. Wallenberg, and L. Samuelson, Nano Lett. 4, 1987 (2004).

${ }^{2}$ K. Tomioka, J. Motohisa, S. Hara, and T. Fukui, Nano Lett. 8, 3475 (2008).

${ }^{3}$ K. Tomioka, Y. Kobayashi, J. Motohisa, S. Hara, and T. Fukui, Nanotechnology 20, 145302 (2009).

${ }^{4}$ J. Noborisaka, J. Motohisa, and T. Fukui, Appl. Phys. Lett. 86, 213102 (2005).

${ }^{5}$ M. A. Olmstead, R. D. Bringans, R. I. G. Uhrberg, and R. Z. Bachrach, Phys. Rev. B 34, 6041 (1986).

${ }^{6}$ R. I. G. Uhrberg, R. D. Bringans, M. A. Olmstead, R. Z. Bachrach, and J. E. Northrup, Phys. Rev. B 35, 3945 (1987).

${ }^{7}$ J. R. Patel, J. A. Golovchenko, P. E. Freeland, and H.-J. Gossmann, Phys. Rev. B 36, 7715 (1987).

${ }^{8}$ R. S. Becker, B. S. Swartzentruber, J. S. Vickers, M. S. Hybertsen, and S. G. Louie, Phys. Rev. Lett. 60, 116 (1988).

${ }^{9}$ R. L. Headrick and W. R. Graham, Phys. Rev. B 37, 1051 (1988).

${ }^{10}$ M. Copel and R. M. Tromp, Phys. Rev. B 37, 2766 (1988).

${ }^{11}$ M. Copel, R. M. Tromp, and U. K. Köhler, Phys. Rev. B 37, 10756 (1988).

${ }^{12}$ R. D. Meade and D. Vanderbilt, Phys. Rev. Lett. 63, 1404 (1989).

${ }^{13}$ C. Cheng and K. Kunc, Surf. Sci. 365, 383 (1996).

${ }^{14}$ C. Cheng and K. Kunc, Z. Phys. B 104, 643 (1997).

${ }^{15}$ E. Kaxiras, Europhys. Lett. 21, 685 (1993).

${ }^{16}$ T. Akiyama, K. Nakamura, and T. Ito, Phys. Rev. B 73, 235308 (2006).

${ }^{17}$ T. Akiyama, K. Nakamura, and T. Ito, Phys. Rev. B 74, 033307 (2006).

${ }^{18}$ F. Glas, J.-C. Harmand, and G. Patriarche, Phys. Rev. Lett. 99, 146101 (2007).

${ }^{19}$ V. G. Dubrovskii and N. V. Sibirev, Phys. Rev. B 77, 035414 (2008).

${ }^{20}$ V. G. Dubrovskii, N. V. Sibirev, J. C. Harmand, and F. Glas, Phys. Rev. B 78, 235301 (2008).
${ }^{21}$ T. Akiyama, Y. Haneda, K. Nakamura, and T. Ito, Phys. Rev. B 79, 153406 (2009).

${ }^{22}$ S. Cahangirov and S. Ciraci, Phys. Rev. B 79, 165118 (2009).

${ }^{23}$ P. Hohenberg and W. Kohn, Phys. Rev. 136, B864 (1964).

${ }^{24}$ J. P. Perdew, K. Burke, and M. Ernzerhof, Phys. Rev. Lett. 77, 3865 (1996).

${ }^{25}$ Y. Morikawa, H. Ishii, and K. Seki, Phys. Rev. B 69, 041403(R) (2004).

${ }^{26}$ I. Hamada and Y. Morikawa, J. Phys. Chem. C 112, 10889 (2008).

${ }^{27}$ S. Yanagisawa, K. Lee, and Y. Morikawa, J. Chem. Phys. 128, 244704 (2008)

${ }^{28}$ H. Koga and T. Ohno, Phys. Rev. B 74, 125405 (2006).

${ }^{29}$ W. Kohn and L. J. Sham, Phys. Rev. 140, A1133 (1965).

${ }^{30}$ N. Troullier and J. L. Martins, Phys. Rev. B 43, 1993 (1991).

${ }^{31}$ K. Takayanagi, Y. Tanishiro, M. Takahashi, and S. Takahashi, J. Vac. Sci. Technol. A 3, 1502 (1985).

${ }^{32}$ P. Mårtensson, G. Meyer, N. M. Amer, E. Kaxiras, and K. C. Pandey, Phys. Rev. B 42, 7230 (1990).

${ }^{33}$ H. B. Elswijk, D. Dijkkamp, and E. J. van Loenen, Phys. Rev. B 44, 3802 (1991).

${ }^{34}$ J. P. Perdew and A. Zunger, Phys. Rev. B 23, 5048 (1981).

${ }^{35}$ K. C. Pandey, Phys. Rev. Lett. 47, 1913 (1981).

${ }^{36}$ As argued in Sec. III A, the energetics of the Si(111)-As surface is mostly governed by As-Si bond orientation. Given the large variations in this orientation, covalent contributions are likely to outweigh vibrational and entropy ones. A similar argument holds for the In-adatom structures examined here, which differ in interlayer covalent interactions (Sec. III B).

${ }^{37}$ E. Kaxiras, Atomic and Electronic Structure of Solids (Cambridge University Press, Cambridge, 2003), p. 199.

${ }^{38}$ J. M. Nicholls, B. Reihl, and J. E. Northrup, Phys. Rev. B 35, 4137 (1987).

${ }^{39}$ For the faulted and unfaulted models, $T_{4}$ is found to be 0.05 and $0.03 \mathrm{eV}$ per reconstruction cell, respectively, lower in energy than $\mathrm{H}_{3}$. 